\title{
Aufsichtsrats- bzw. Diversitätsmerkmale und Profitabilität von deutschen und österreichischen Versicherungsunternehmen
}

\author{
Helmut Pernsteiner · Eva Wagner
}

Online publiziert: 27. Oktober 2017

(C) Der/die Autor(en) 2017. Dieser Artikel ist eine Open-Access-Publikation.

Zusammenfassung Im Rahmen der allgemeinen Corporate Governance Diskussion fokussiert dieser Beitrag auf das Organ Aufsichtsrat und geht der Frage eines Zusammenhanges von Eigenschaften des Aufsichtsrates (Mitgliederzahl und -zusammensetzung, Sitzungszahl, Geschlecht, Entlohnung und Kapitalmarktorientierung) und der Profitabilität anhand der größten deutschen und österreichischen Versicherungsgesellschaften nach.

Es zeigen sich - vor allem größenbedingt - unterschiedliche Ergebnisse in den beiden untersuchten Ländern. In der empirischen Analyse können u. a. folgende Aspekte als statistisch signifikant erkannt werden: Die Unternehmensgröße sowie die Börsennotiz sind mit Diversitätsaspekten bzw. dem Frauenanteil positiv korreliert, ebenso steigt die Entlohnung der Kontrollorgane stark mit der Größe der Versicherungsgesellschaft an. Es zeigt sich - bis auf die Sitzungshäufigkeit - kein Zusammenhang zwischen den untersuchten Merkmalen sowie dem Ausmaß des Diversitätsmanagements und der Performance der Assekuranzen.

\section{Board characteristics and profitability for German and Austrian insurance companies}

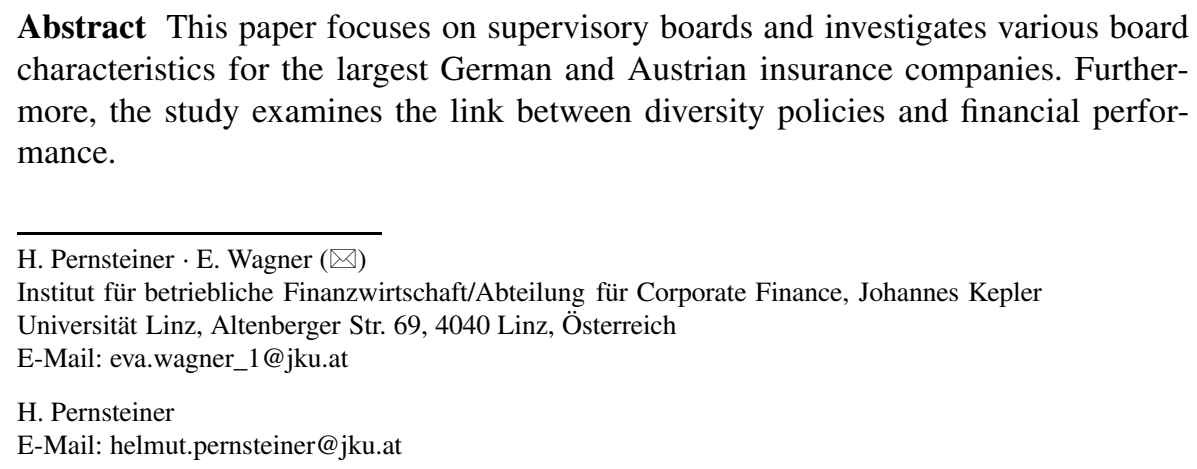

Abstract This paper focuses on supervisory boards and investigates various board characteristics for the largest German and Austrian insurance companies. Furthermore, the study examines the link between diversity policies and financial performance.

H. Pernsteiner · E. Wagner $(\bowtie)$

Institut für betriebliche Finanzwirtschaft/Abteilung für Corporate Finance, Johannes Kepler Universität Linz, Altenberger Str. 69, 4040 Linz, Österreich

E-Mail: eva.wagner_1@jku.at

H. Pernsteiner

E-Mail: helmut.pernsteiner@jku.at 
The study reveals some differences between the two aforementioned countries, which are mainly driven by size effects. In addition, the analysis shows that larger and stock listed insurance companies have better diversity management policies and a higher proportion of female directors. There is no significant correlation between gender diversity measures and profitability.

\section{Einleitung}

Die thematische Welle der Corporate Governance, die ihren Ursprung in angelsächsischen Ländern hatte, hat längst Kontinentaleuropa erreicht und ist zum intensiven Diskussionsgegenstand in den Unternehmen und v. a. in den Rechts- und Wirtschaftswissenschaften geworden. Corporate Governance - hier verstanden als (guter) Ordnungsrahmen für die Leitung und Überwachung von Unternehmen - fand als Themenbereich zuerst Aufnahme in den Managementebenen der börsennotierten Aktiengesellschaften, zumal auch der Kapitalmarkt höchstes Interesse hatte; ging und geht es doch letztlich darum, Unternehmen mit einer guten Corporate Governance zu identifizieren und dann dies als Ausgangspunkt zu nehmen, Unternehmen mit höherer Profitabilität bzw. Performance ausfindig zu machen. Corporate Governance ist damit kein Selbstzweck, sondern zielt darauf ab, dass Unternehmen, wo dieser Ordnungsrahmen ,besser und effizienter“ funktioniert, letztlich profitabler, stärker mit ihren Eigenkapitaltiteln nachgefragt sind und damit höhere Bewertungen der gehandelten Anteile (Aktien) aufweisen.

Das Themenfeld Corporate Governance ist jedoch breit und uneinheitlich. Die Uneinheitlichkeit lässt sich an den primär länderweise organisierten Corporate Governance Codices ablesen, wo Unterschiede zwischen dem stärker Shareholder Valueorientierten angelsächsischen Regelungsrahmen und den auch andere Stakeholder im Blickfeld habenden primär kontinentaleuropäischen Kodifikationen erkennbar sind. Die Breite wiederum lässt sich an den Organen wie Vorstand, Aufsichtsrat und Hauptversammlung (in der Diktion der Aktiengesellschaft) sowie Abschlussprüfer und Revision erkennen.

Dieser Beitrag hat den Aufsichtsrat im Visier. Dieser ist ein ganz überwiegend von den Herkünften der Mitglieder heterogenes Organ, das seinen Namen entsprechend primär Überwachungsaufgaben zu bewältigen hat, aber auch durch den Einfluss auf die operative Leitungsebene (v. a. Bestellung des Vorstands) bekanntermaßen zentral für das Unternehmen ist.

Selbstverständlich hat man sich schon früher - natürlich auch schon vor der angelsächsisch motivierten Corporate Governance Diskussion - den Kopf darüber zerbrochen, wie durch Unabhängigkeit, fachliche Qualität der Mitglieder und durch organisatorische Maßnahmen (z. B. Sitzungszahlen) die Wirkung und Effizienz dieses Organs verbessert werden kann. ${ }^{1}$ Auch das Aktienrecht hat sich verändert und diesem Trend Rechnung getragen. Es liegt folglich die Frage nicht weit entfernt, wieweit Maßnahmen zur Verbesserung der Unabhängigkeit und Qualität des Aufsichtsrats die Profitabilität bzw. Performance tatsächlich verbessert haben. Dies ist

1 Vgl. Kalss und Schimka (2016), S. 77 ff. 
Tab. 1 Rechtsformen der Versicherungsunternehmen in D 2009-2013

\begin{tabular}{llllll}
\hline & 2009 & 2010 & 2011 & 2012 & 2013 \\
\hline VVaG (ausgenommen kleine VVaG) & 79 & 78 & 78 & 77 & 77 \\
Kleine VVaG & 23 & 23 & 23 & 23 & 23 \\
AG & 278 & 274 & 276 & 267 & 259 \\
SE & - & - & - & - & 4 \\
Gesamt & 380 & 375 & 377 & 367 & 363 \\
\hline
\end{tabular}

Quelle: in Anlehnung an BaFin, www.bafin.de

in dieser Breite wohl schwierig zu messen, bei einzelnen Aspekten wird hier der Versuch unternommen Einflüsse auf die Profitabilität festzustellen oder zu verneinen.

In diesem Beitrag werden die Aspekte Größe des Aufsichtsrats (Zahl der Mitglieder), Diversity (hier dabei insbesondere das Geschlecht) im Aufsichtsrat, Sitzungsanzahl, Entlohnung der Mitglieder des Aufsichtsrats und die Kapitalmarktorientierung für Eigenkapital (Börsennotiz) einer Prüfung unterzogen. Selbstverständlich müssen bei einer quantitativen empirischen Untersuchung Fokussierungen erfolgen: Dies geschieht einmal mit der Ausrichtung auf Versicherungsunternehmen auf eine klar definierte Branche, für die spezifische Governance-Untersuchungen - trotz der ökonomischen Bedeutung - spärlich sind; ferner auf Versicherungsunternehmen, die ihren Sitz in Deutschland und Österreich haben (aufgrund des in der Schweiz gültigen Board Systems wurde die Schweiz in diese vergleichende Diskussion deutschsprachiger Staaten nicht einbezogen); damit erfolgt ein Vergleich zwischen zwei Ländern mit relativ ähnlicher rechtlicher Grundlage, aber mit unterschiedlichen Größenordnungen und ggf. differierenden Landeskulturen. Für das Jahr 2013 können in Österreich 100 Versicherungsunternehmen (davon 41 Aktiengesellschaften), in Deutschland 363 Versicherungsunternehmen (davon 259 Aktiengesellschaften) festgestellt werden (vgl. Tab. 1 für Deutschland). Dies alleine ist schon ein Indiz für unterschiedliche Größenordnungen.

Die Aufarbeitung des skizzierten Themenbereiches gestaltet sich wie folgt: Im nächsten Kapitel wird Corporate Governance einer Analyse unterzogen, dann erfolgt der Bezug auf Versicherungsunternehmen. Nach dieser Diskussion werden die angesprochenen Aspekte des Aufsichtsrats diskutiert, um im Kap. 5 die empirische Erhebung zu zeigen. Anschließend erfolgt eine Analyse der Ergebnisse und der Abschluss als Fazit mit einem Ausblick.

\section{Corporate Governance und Aufsichtsrat}

Basis des hier vertretenen Verständnisses von Corporate Governance ist die Sichtweise als „Ordnungsrahmen für die Leitung und Überwachung eines Unternehmens“. Als theoretische Basis für Corporate Governance fungieren die PropertyRights-, Transaktionskosten-, Prinzipal-Agenten-Theorie und die Stewardshiptheorie. ${ }^{2}$ Die letzteren beiden sind aufgrund der Ausrichtung auf den Aufsichtsrat von

\footnotetext{
2 Vgl. Welge und Eulerich (2012), S. 7 ff.
} 
besonderer Bedeutung, da der Aufsichtsrat überwiegend (s. Arbeitnehmermitbestimmung) als Agent der die Eigentümerschaft verkörpernden Hauptversammlung tätig wird und sich damit die Eigeninteressen der Aufsichtsratsmitglieder negativ auf die Performance, die im zentralen Interesse der Eigentümer steht, auswirken können, weil z. B. Aufsichtsratsmitglieder nicht unabhängig gegenüber dem Vorstand sind oder ihre Aufgaben mit mangelndem Engagement wahrnehmen. Entgegen kann die Stewardship-Theorie zeigen, wo gerade diese Aufsichtsratsmitglieder z. B. aufgrund ihres hohen Interesses ihre Aufgabe mit höchstem Einsatz durchführen. Die Ambivalenz kann am Beispiel des langjährigen Vorstandsvorsitzenden, der aufgrund seiner Erfahrungen, Kontakte und seines Wissens in den Aufsichtsrat gewählt wird (vielleicht sogar zum Vorsitzenden), gezeigt werden. Seine Verbundenheit mit dem Unternehmen und sein hohes Engagement soll sich positiv auswirken (Stewardship-Überlegung). Dagegen kann jedoch vorgebracht werden, dass er die Überwachungsaufgabe aufgrund seiner früheren Entscheidungen und Maßnahmen nur eingeschränkt wahrnehmen kann, da eine massive Kritik an diesen hochwahrscheinlich ausgeschlossen werden kann (Prinzipal-Agenten-Theorie). Die Gesetzgeber und die Codices haben wohl letzteres Argument als schwerwiegender empfunden, sind also nach agententheoretischen Überlegungen vorgegangen.

Sowohl das Aktiengesetz (AktG) als auch der Corporate Governance Kodex (CGK) haben durchaus eine Stärkung des Aufsichtsrats im Auge (gehabt), indem die fachliche Qualität der Aufsichtsratsmitglieder, eine ausgewogene Zusammensetzung des Organs, ein ausreichendes zeitliches Engagement (z. B. über die Begrenzung der Mandate) und die Unabhängigkeit und Freiheit von Interessenskonflikten direkt bzw. indirekt thematisiert wird. ${ }^{3}$

Durchgerungen hat man sich zu einem Druck zur intensiveren thematischen Auseinandersetzung über eine verstärkte Ausschussarbeit, zur Thematisierung der Vergütungsfrage ohne konkrete Vorgaben, zur Verschärfung der Unabhängigkeitsbestimmungen und zur verstärkten Transparenz des Wahlprocederes. ${ }^{4}$ Schwierig bis unmöglich ist natürlich eine konkrete Präzisierung von Qualifikationsanforderungen, da eine Festlegung von formalen Qualifikationsanforderungen (z. B. akademische Studien) erfolgreiche und damit qualifizierte Unternehmerpersönlichkeiten ausschließen könnte, womit man dem Organ Aufsichtsrat keinen Dienst erweisen würde. Kalss und Schimka weisen jedoch darauf hin, dass Grundkenntnisse der gesetzlichen Rahmenbedingungen und Kenntnisse in betriebswirtschaftlichen Grundfragen und über das Funktionieren von Marktmechanismen vorhanden sein müssen, ferner das Lesen von Jahresabschlüssen und ihre Analyse und die kritische Hinterfragung von Berichten des Vorstands möglich sein sollten. ${ }^{5}$ Allerdings tauchen konkreter die Argumente Diversität (auf das Geschlecht bezogen), Altersstruktur und Internationalität auf. Bei der Problematik einer oftmaligen Wiederwahl eines Aufsichtsratsmitglieds ist man jedoch hinsichtlich einer Regelung zurückhaltend,

\footnotetext{
3 Vgl. Guserl und Pernsteiner (2015), S. 46; für Kreditinstitute gelten bspw. weitere persönliche Voraussetzungen der Aufsichtsratsmitglieder siehe Hölscher und Dähne (2014), S. 285 f.

4 Vgl. Pernsteiner (2015), S. 195 ff.

5 Vgl. Kalss und Schimka (2016), S. 97.
} 
obwohl dies im internationalen Umfeld durchaus vorkommt. ${ }^{6}$ Manchmal wird aber eine kürzere Bestelldauer als die im Aktienrecht mögliche vorgeschlagen. ${ }^{7}$ Hier ist eine ähnliche Ambivalenz wie beim obigen Beispiel des Vorstandsvorsitzenden, der zum Aufsichtsrat(spräsidenten) gewählt wird, feststellbar: Für eine langjährige Aufsichtsratstätigkeit (mit entsprechenden Wiederwahlen) spricht die zunehmende Erfahrung und die anzunehmende wachsende (auch emotionale) Verbundenheit mit dem Unternehmen, was auf die Stewardshiptheorie verweist, dagegen die Betriebsblindheit, eine das Engagement zunehmend behindernde persönliche Verflechtung mit möglichem Blick auf Eigeninteressen; letzteres kann agententheoretisch begründet werden. Im Gegensatz zum obigen Fall des Vorstandsvorsitzenden wird dies nicht thematisiert, lediglich indirekt über die Altersstruktur, wo eine Ausgewogenheit im Aufsichtsrat anzustreben ist.

Damit wird ersichtlich, dass Corporate Governance-Themen im Organ Aufsichtsrat angekommen sind, die zu Änderungen der Vorgaben und damit auch der realen Situation geführt haben.

Etwas anders verhält es sich mit der Frage nach der Diversität. Die Diskussion nahm ihre Entwicklung nicht aus der Corporate Governance-Debatte, sondern aus dem allgemeinen gesellschaftlich-politischen Diskurs und fand u. a. auch Eingang in das Organ Aufsichtsrat, wo - wie in vielen Führungsgremien der Wirtschaft eine Männerdominanz zu konstatieren war und ist.

Diversität kann am besten mit den Begriffen Heterogenität bzw. Vielfalt umschrieben werden. ${ }^{8}$ Als Kerndimensionen haben sich das Alter, das Geschlecht, die Herkunft, die sexuelle Orientierung, Behinderungen, Religion und Rasse herauskristallisiert. ${ }^{9}$ In Zusammenhang mit der Analyse des Aufsichtsrats wird primär das Thema Geschlecht (männlich/weiblich) verfolgt, ggf. wäre auch das Alter der Mitglieder des Aufsichtsrats interessant. Es - das Geschlecht - handelt sich damit um die wahrnehmbaren Faktoren von Diversität ${ }^{10}$ und gehört nach Gardenswartz und Rowe (2003) zum innersten Kern ihres Klassifizierungsmodells („Persönlichkeit des Menschen“"). ${ }^{11}$ Die Bedeutung des Geschlechts im Feld Diversität ist damit unstrittig. Ferner wird Diversität als Ressource für den Unternehmenserfolg angesehen. ${ }^{12}$ Der Anteil von Frauen in den Aufsichtsräten ist Untersuchungen nach gering. ${ }^{13}$ Die auch sonst angeführten Gründe der schwierigen Vereinbarkeit von Familie und Beruf und die geringere Präsenz in für eine Aufsichtsratstätigkeit bedeutsamen Netzwerken können als Gründe genannt werden. ${ }^{14}$

Allgemein kann festgehalten werden, dass Untersuchungen und Überlegungen hinsichtlich der Aspekte des Aufsichtsrats und der Profitabilität der Unternehmen

\footnotetext{
6 Vgl. Pernsteiner (2015), S. 196.

7 Vgl. Koeberle-Schmid (2012), S. 135 f.

8 Vgl. Mensi-Klarbach (2010), S. 23 und Simon (2002), S. 359.

9 Vgl. Krell et al. (2007).

10 Vgl. Sepheri (2002).

11 Vgl. Gardenswartz und Rowe (2003).

12 Vgl. Süß und Kleiner (2006), S. 58.

13 s. dazu bspw. die Untersuchung von Holst und Kirsch (2014).

14 Vgl. Kalss (2011).
} 
den Vergleich unterschiedlicher (Sitz-)Staaten im Blickpunkt haben bzw. - alleine aufgrund der Datenverfügbarkeit - auf börsennotierte und/oder große Unternehmen ausgerichtet sind. Branchenaspekte treten nicht so stark hervor. In diesem Beitrag erfolgt eine Fokussierung auf Versicherungsunternehmen. Für diese liegen - nach Kenntnis der Autoren - im deutschsprachigen Raum keine diesbezüglichen Untersuchungen vor.

\section{Corporate Governance und Aufsichtsrat von Versicherungsunternehmen}

Bislang lag der Diskussion stillschweigend als „Musterrechtsform“ die Aktiengesellschaft zugrunde, die zweifellos in der Versicherungsbranche eine große Rolle spielt. Bekanntermaßen sind auch die Europäische Gesellschaft (SE) und der Versicherungsverein auf Gegenseitigkeit (VVaG) mögliche Rechtsformen.

Im sog. großen $V V a G$ (nur dieser ist aufgrund des Samples für die empirische Erhebung interessant) ist ebenfalls ein Aufsichtsrat notwendig. Wird eine (Versicherungs-)SE als Rechtsform gewählt, so kann zwischen dem in Deutschland und Österreich gültigen dualistischen System, wo sich die Struktur wie bei der Aktiengesellschaft darstellt, und dem angelsächsischen monistischen System gewählt werden. Zur Übersicht siehe Tab. 2.

Der besonderen Situation von Versicherungsunternehmen wird dann noch durch einige Sondernormen Rechnung getragen. So wird eine fachliche Qualifikation (ausreichende Berufsqualifikationen, Kenntnisse und Erfahrungen) und eine persönliche Zuverlässigkeit von den Mitgliedern des Aufsichtsrats gefordert. ${ }^{15}$ In Deutschland kann die Aufsichtsbehörde BaFin eine Verwarnung bei Nichteinhaltung der erforderlichen Qualifikation für Aufsichtsratsmitglieder aussprechen, zudem die Abberufung verlangen und eine Tätigkeitsuntersagung aussprechen. ${ }^{16}$

Die Entlohnung muss das Erfordernis erfüllen, angemessen, transparent und nachhaltig zu sein, wobei die Vergütung nicht mit der Vermittlung von Versicherungsverträgen zusammenhängen darf. ${ }^{17}$

Durchaus immer wieder im Blickpunkt ist die Frage der Unabhängigkeit der Mitglieder - trotz der Problematik der Definition. Hier erfolgen Regelungen über die Corporate Governance Codices.

Ein Zusammenhang mit der Qualität der Überwachung könnte sowohl mit der Größe des Aufsichtsorgans (Anzahl der Mitglieder) als auch mit der Anzahl der Sitzungen des Aufsichtsrats hergestellt werden. Hier wird in Deutschland für die Größe des Aufsichtsrats eine Bandbreite von 3-21 natürlichen Personen, in Österreich eine solche von 3-20 (zuzüglich der Arbeitnehmervertreter) normiert. Diese Regelungen sind nicht versicherungstypisch.

\footnotetext{
15 Vgl. § 123 Abs 1 VAG.

16 Vgl. Kalss und Wendt (2016), S. 1296.

17 Vgl. Eling und Pankoke (2014), S. 148 ff.
} 
Tab. 2 Übersicht über die Rechtsformunterschiede

\begin{tabular}{|c|c|c|c|}
\hline & Versicherungs-AG & Versicherung-SE & Großer VVaG \\
\hline $\begin{array}{l}\text { Abgrenzungs- } \\
\text { merkmale }\end{array}$ & \multicolumn{2}{|c|}{ Kapitalhingabe (Kapitalverein) } & $\begin{array}{l}\text { Personale Elemente } \\
\text { (Personenverein) }\end{array}$ \\
\hline \multirow[t]{2}{*}{ Ziel/e } & \multicolumn{2}{|l|}{ Gewinnerzielung } & Bedarfsdeckung \\
\hline & \multicolumn{3}{|c|}{ Unternehmenswertsteigerungen } \\
\hline \multirow[t]{8}{*}{ Organe (zwingend) } & Vorstand & Monistisches System & Vorstand \\
\hline & Aufsichtsrat & Verwaltungsrat & Aufsichtsrat \\
\hline & \multirow[t]{6}{*}{ Hauptversammlung } & $\begin{array}{l}\text { Geschäftsführende } \\
\text { Direktoren }\end{array}$ & \multirow{6}{*}{$\begin{array}{l}\text { Oberstes Organ (Mit- } \\
\text { gliederversammlung, } \\
\text {-vertretung) }\end{array}$} \\
\hline & & Dualistisches System & \\
\hline & & Vorstand & \\
\hline & & Aufsichtsrat & \\
\hline & & Für beide & \\
\hline & & Hauptversammlung & \\
\hline \multirow{2}{*}{$\begin{array}{l}\text { Corporate } \\
\text { Governance-Kodex }\end{array}$} & \multicolumn{2}{|c|}{ Zwingend für börsennotierte $\mathrm{AG}$ und $\mathrm{SE}$} & Freiwillig \\
\hline & \multicolumn{2}{|c|}{ Freiwillig für alle anderen } & \\
\hline
\end{tabular}

\section{Untersuchungsaspekte des Aufsichtsrats}

Ein Schwerpunkt der Untersuchung wird in der Größe des Aufsichtsrats (Mitgliederzahl) liegen. Wie eben erwähnt ist diese - nicht versicherungstypisch - begrenzt, wobei hier nur die Begrenzung nach oben interessant ist. Das Argument für eine Höchstbegrenzung liegt in einer befürchteten Schwerfälligkeit, was eine langsame Entscheidungsfindung bedeuten kann. ${ }^{18}$ Besonders hinsichtlich der zustimmungspflichtigen Geschäfte könnte eine langsame Entscheidungsfindung nachteilig sein; insgesamt wäre argumentativ zumindest plausibel, dass große Aufsichtsräte mit mangelnder Profitabilität einhergehen. Beispielhaft erklärt die Allianz SE 2007: „Ein bedeutender Fortschritt ist außerdem die deutliche Verkleinerung des Aufsichtsrates von 20 auf zwölf Mitglieder. Die arbeitsfähige Größe ist die Herausforderung des deutschen Aufsichtsratssystems - zu große Gremien gehen zu Lasten von Effizienz und Vertraulichkeit."19

Empirische (nicht versicherungsspezifische) Untersuchungen zeigen jedoch ein nicht so eindeutiges Bild: Zur Aussage, dass eher kleinere Boards - fast alle Studien basieren auf dem angelsächsischen Board-Modell - zu einem höheren Unternehmenswert und damit zu einer höheren Unternehmensperformance führen teilen Yermack (1996), Eisenberg et al. (1998) und Cheng (2008), während Bhagat und Black (2002) und Bermig und Frick (2011) - letztere für deutsche Unternehmen einen signifikanten Einfluss nicht nachweisen konnten. ${ }^{20}$ Dalton et al. (1998) kön-

\footnotetext{
18 Vgl. Eling und Pankoke (2014), S. 158; so weisen auch Hüffer und Koch (2016), § 95 Rn 1, darauf hin, dass durch Höchstzahlen die Effektivität des Aufsichtsrats verbessert wird; Drygala (2015b), § 95 Rn 1, zweifelt dies hingegen an.

19 Vgl. Allianz SE (2007), o.S.

20 Vgl. Yermack (1996); Eisenberg et al. (1998); Cheng (2008); Bhagat und Black (2002); Bermig und Frick (2011).
} 
nen in ihrer umfangreichen Metastudie - allerdings eben schon etwas älter - keinen Zusammenhang zwischen der Unternehmensperformance und der Board-Zusammensetzung erkennen. Problematisch in diesem Zusammenhang ist, dass die Zahl der Aufsichtsratsmitglieder i. d. R. mit der Unternehmensgröße zusammenhängt. ${ }^{21}$

Die Diversität des Aufsichtsrats stellt ein ebenso spannendes Untersuchungsfeld dar, zumal besonders der Frauenanteil im politischen und gesellschaftlichen Diskurs steht und ebenso wie die Internationalität teilweise in den gesetzlichen Grundlagen, vor allem jedoch in den Corporate Governance Codices vorkommen. Die Norm des $\S 96$ Abs 2 und 3 AktG sieht für Neuwahlen und Entsendungen ab 01.01.2016 vor, dass v. a. für börsennotierte Aktiengesellschaften ein Frauenanteil von mind. $30 \%$ sukzessive zu erfüllen ist. ${ }^{22}$ Das österreichische Recht kennt eine solche Norm nicht, es wird in $\S 87$ Abs. 2a öAktG lediglich auf die Diversität und Internationalität verwiesen. ${ }^{23}$ Relevant ist für börsennotierte Versicherer auch der Corporate Governance Kodex; in Deutschland empfiehlt der DCGK seit 2010, konkrete Ziele für die angemessene Beteiligung von Frauen in den Aufsichtsräten zu formulieren (DCGK 2010). In Österreich sieht der Kodex seit 2012 verpflichtend vor, dass Aspekte der Diversität des Aufsichtsrats (auch) im Hinblick auf die Vertretung beider Geschlechter angemessen zu berücksichtigen sind. Zusätzlich ist gem. $§ 243 \mathrm{c}$ Abs. 2 Z 2 UGB ist ab dem Geschäftsjahr 2010 verpflichtend im Corporate Governance Bericht anzuführen: ,,... welche Maßnahmen zur Förderung von Frauen im Vorstand, im Aufsichtsrat und in leitenden Stellungen ( 80 AktG) der Gesellschaft gesetzt wurden.“

Auf eine Qualifikationsanforderung wird grundsätzlich verzichtet, lediglich nach $\S 100$ Abs 5 AktG muss mind. ein Aufsichtsratsmitglied sachverständig auf den Gebieten Rechnungslegung und Abschlussprüfung sein.

Frauen kommen in Deutschland und Österreich in den Aufsichtsräten in geringem Ausmaß vor; es gibt eine erhebliche Zahl von börsennotierten Unternehmen, die überhaupt keine Frau in diesem Gremium aufweisen. ${ }^{24}$ Da sich die empirische Analyse auf einen Zeitraum bis einschließlich 2013 bezieht, ist die oben genannte gesetzliche Vorgabe noch nicht relevant, zeigt aber möglicherweise den Handlungsbedarf auf und kann ggf. nachträglich zur Diskussion der Vorteilhaftigkeit dieser Aufgabenstellung herangezogen werden.

Ähnlich verhält es sich mit der Internationalität, wobei hier bereits die Definition des internationalen Aufsichtsratsmitglieds unklar ist und primär auf das Gebiet der hauptberuflichen Tätigkeit abgestellt werden sollte. Diese Thematik genießt in angelsächsisch ausgerichteten Unternehmen höhere Priorität und wird wahrscheinlich einen Bezug zur geografischen Geschäftstätigkeit, Größe und Eigentümerschaft des Unternehmens aufweisen.

\footnotetext{
21 Vgl. Dalton et al. (1998).

22 Vgl. Hüffer und Koch (2016), § 96 Rn 13; Drygala (2015b), § 96 Rn 32 und 36 ff. weist darauf hin, dass es sich um eine sozialpolitische Maßnahme handelt und trägt verfassungs- und europarechtliche Bedenken vor.

23 Vgl. Kalss und Schimka (2016), S. 93.

24 Vgl. Holst und Kirsch (2014), S. 28.
} 
Neben der gesellschaftspolitischen Bedeutung interessiert Diversität auch in ökonomischer Perspektive, also ob Diversität zum Unternehmenserfolg beiträgt.

Argumentiert wird hier primär damit, dass Frauen einen anderen Führungsstil pflegen, eine größere Perspektivenvielfalt einbringen und in der Folge Kreativität, Ideen, Innovationskraft - insgesamt das Humankapital - gesteigert und Wettbewerbsvorteile generiert werden können. ${ }^{25}$ Diversität kann auch zu einem besseren Image und höherer Reputation des Unternehmens führen, was sich positiv auf das Verhalten der Kunden auswirkt. ${ }^{26}$ Insgesamt führt „Diversity-Leadership“ somit in dieser Argumentation zu höherer Performance. Argumente, die gegen die Unternehmenswertsteigerung sprechen sind etwa, dass heterogene(re) Boards weniger effizient bei der Kommunikation und im Entscheidungsfindungsprozess sind. ${ }^{27}$

Insbesondere im angloamerikanischen Raum wurde zum Thema Diversität und Unternehmenswert bzw. Performance eine Vielzahl von Studien publiziert, welche insgesamt gemischte Evidenz liefern. In einer Studie aus 2014 kommen McKinsey \& Company hinsichtlich Diversity-Management in Industrieunternehmen zum Ergebnis, dass bei hoher Gender Diversity in der Führung der Unternehmen mit einer um $15 \%$ höheren Wahrscheinlichkeit ausgegangen werden kann, dass die Profitabilität über dem nationalen Durchschnitt liegt (McKinsey \& Company 2014). Zu den meistzitierten wissenschaftlichen Arbeiten zählt die Studien von Adams und Ferreira (2009). ${ }^{28}$ Sie finden in ihrer großangelegten Untersuchung für die USA (Zeitraum 1996 bis 2003), dass die Präsenz weiblicher Board-Mitglieder positive Governance-Effekte, wie bspw. Unabhängigkeit, verbesserte Monitoring-Tätigkeit, höhere Anwesenheit bei Sitzungen hat und mit spezifischen Entlohnungscharakteristika einhergeht (bspw. höhere aktienbasierte Vergütung). Jedoch liefert die Untersuchung keine Hinweise darauf, dass ein höherer Frauenanteil kausal zu besserer Performance führt.

Als beeinflussende Faktoren für die Präsenz von Frauen bzw. den Frauenanteil in Boards konnte in der Literatur vor allem die Unternehmensgröße und die Größe des Boards identifiziert werden. ${ }^{29}$ Auch die Branche spielt eine Rolle. ${ }^{30}$ Es kann argumentiert werden, dass gerade in Dienstleistungsbranchen, in denen der Anteil der weiblichen Belegschaft hoch ist, Frauen entsprechend stärker im Board repräsentiert sind. ${ }^{31}$ Bezogen auf die Versicherungswirtschaft sind nach Kenntnis der Autoren keine Studien verarbeitet. Häufig wird in der Empirie sogar der gesamte Finanzsektor exkludiert. $^{32}$

Wie bereits erwähnt, soll durch die ständige Auseinandersetzung des Aufsichtsrats mit den unternehmerischen Themen die Qualität der Überwachung verbessert

\footnotetext{
25 Vgl. Watson et al. (1993), S. 590 ff; Erhardt et al. (2003), S. 103; Carter et al. (2003), S. 36; Adams und Ferreira (2009), S. 305; Heidemann et al. (2013), S. 7 f.

26 Vgl. Smith et al. (2006), S. 571.

27 Vgl. Carter et al. (2003), S. 36; Adams und Ferreira (2009), S. 305.

$28 \mathrm{Vgl}$. Adams und Ferreira (2009), S. $291 \mathrm{ff}$.

29 Vgl. Adams und Ferreira (2009); Carter et al. (2003).

30 Vgl. Harrigan (1981).

31 Vgl. Farrell und Hersch (2005), S. 89.

32 Vgl. bspw. Hermalin und Weisbach (1991) sowie Farrell und Hersch (2005).
} 
werden. Die Festlegung einer Mindestsitzungszahl soll dem Rechnung tragen: in der Schweiz etwa wird dies nicht reguliert. ${ }^{33}$ Bei börsennotierten Gesellschaften muss der Aufsichtsrat zwei Sitzungen im Halbjahr abhalten, bei nicht börsennotierten Gesellschaften kann er sich auf eine im Halbjahr einigen. ${ }^{34}$ Dies soll die Kontrollfunktion des Aufsichtsrats besser ermöglichen. ${ }^{35}$ Ähnlich gelagert ist § 94 Abs 3 öAktG, der mindestens eine vierteljährliche Sitzungsfrequenz für alle Aktiengesellschaften vorschreibt. Kalss (2012) betont den Charakter einer Mindestzahl, wo wenn notwendig - die Zahl der Sitzungen zu erhöhen ist. ${ }^{36}$ Koeberle-Schmid (2012) empfiehlt dabei eine Sitzungsdauer von vier Stunden. ${ }^{37}$

Interessanterweise zeigen empirische Studien diesbezüglich eher in das Gegenteil. Vafeas (1999) kommt zum eher logischen Schluss, dass die Sitzungszahl positiv mit der Board-Größe korreliert ist. Die Board-Größe wird wohl einen positiven Zusammenhang mit der Unternehmensgröße aufweisen. Er kommt jedoch zum Ergebnis, dass eine negative Beziehung zwischen Aufsichtsratssitzungen und dem Unternehmenswert besteht. ${ }^{38}$ Horváth und Spirollari (2012) stellen fest, dass zwischen der Zahl der Aufsichtsratssitzungen und der Performance kein Zusammenhang besteht. ${ }^{39}$

Empirische Studien sind sehr stark auf börsennotierte Unternehmen (primär im angelsächsischen Raum) ausgerichtet. Dies entspricht nicht völlig der mitteleuropäischen Struktur, da zum einen die Versicherungsvereine auf Gegenseitigkeit durchaus präsent sind, zum anderen Eigentümerschaften (Konzerneinbau und genossenschaftsähnliche Strukturen) vorliegen, die nicht zu einer Börsennotiz passen. Diese Eigentümerschaften werden ggf. zu einer anderen Struktur des Aufsichtsrats führen. Dies kann sowohl die personelle Zusammensetzung als auch die inhaltliche Aufgabenwahrnehmung betreffen. Auf die intensivere, verpflichtende und freiwillige Informationsabgabe dieser kapitalmarktorientierten Unternehmen sei verwiesen. Somit ist es von Interesse, auch Merkmale von börsennotierten Versicherungsgesellschaften zu untersuchen.

Schließlich soll noch die Entlohnung der Aufsichtsratsmitglieder untersucht werden. Das AktG ermöglicht die Vergütung für Aufsichtsratsmitglieder, die allerdings angemessen sein soll. ${ }^{40}$ Damit sind feste und/oder variable Vergütungen möglich. Die österreichische Regelung in $\S 98$ öAktG ist ähnlich. ${ }^{41}$ Selbstverständlich bestehen hinsichtlich der Höhe durchaus Unterschiede, wobei insbesondere ein Zusammenhang zur Größe des Unternehmens belegt ist. ${ }^{42}$ Bei Versicherungsunternehmen wird nach $\S 64$ b Abs 1 VAG neben der Angemessenheit auch die Transparenz und die

\footnotetext{
33 Vgl. Eling und Pankoke (2014), S. 156.

34 Vgl. § 110 Abs 3 AktG.

35 Vgl. Drygala (2015a), § 110 Rn 1.

36 Vgl. Kalss (2012), § 94 Rn $26 \mathrm{ff}$.

37 Vgl. Koeberle-Schmid (2012), S. 136.

38 Vgl. Vafeas (1999).

39 Vgl. Horváth und Spirollari (2012).

40 Vgl. § 113 Abs 1a AktG.

41 s. dazu Strasser (2010), §§ 98, 99 Rn. $11 \mathrm{ff}$.

${ }^{42}$ Vgl. Evers (2009), S. 377.
} 
Ausrichtung auf eine nachhaltige Entwicklung gefordert; dies ergibt sich aus der langfristigen Ausrichtung der Versicherungswirtschaft.

\section{Empirische Untersuchung}

\subsection{Datenlage und deskriptive Statistik}

Basis der Untersuchung der genannten Fragestellungen sind die jeweils zehn größten Versicherungsunternehmen (gemessen anhand der Bruttobeitragseinnahmen im Jahr 2013) in Deutschland und in Österreich. Für diese Versicherungsgesellschaften ist die Datenlage im Untersuchungszeitraum als gut zu bezeichnen. Analysiert wurden die Geschäftsjähre 2009-2013. Die zehn größten Unternehmen repräsentieren dabei die österreichische Versicherungsbranche sehr gut, sie haben 2013 einen Marktanteil von über $70 \%$ des gesamten inländischen Marktes (gemessen anhand der verrechneten Prämien). ${ }^{43}$ In Deutschland hatten die Top-Ten-Erstversicherer im Jahr 2013 kumuliert einen Marktanteil von $56 \%$ an den Brutto-Beiträgen. ${ }^{44}$ Dies zeigt die hohe Konzentration im Versicherungsmarkt.

In einer Rechtsformbetrachtung ergaben sich für Deutschland 7 AGs, 1 VVaG und 2 Unternehmen in der Rechtsform einer SE (siehe dazu sowie zu den folgenden Ausführungen auch Tab. 3). In Österreich firmieren die größten Versicherungsunternehmen alle in der Rechtsform einer Aktiengesellschaft. In der Stichprobe sind drei deutsche und zwei österreichische Unternehmen börsennotiert.

In Österreich überwiegen traditionell die Kompositversicherer; als solche werden hier jene bezeichnet, die verschiedene bzw. (auch) die Zweige der Schaden- bzw. Un-

Tab. 3 Überblick über die Geschäftstätigkeit

\begin{tabular}{lll}
\hline Rechtsform der Versicherungsgesellschaften & Deutschland & Österreich \\
\hline AG & 7 & 10 \\
VVaG & 1 & 0 \\
SE & 2 & 0 \\
Börsennotierte Gesellschaften & 3 & 2 \\
Betriebene Sparten & & \\
Krankenversicherung & 5 & 5 \\
Lebensversicherung & 7 & 7 \\
Schaden-/Unfallversicherung & 5 & 10 \\
Allg. Versicherungsgeschäft & 4 & 1 \\
Zuordnung gesamt & & \\
Kompositversicherer & 3 & 10 \\
Lebens- bzw. Krankenversicherer & 5 & 0 \\
Rückversicherer & 2 & 0 \\
\hline
\end{tabular}

43 Vgl. Versicherungsverband Österreich (2013), S. 6.

44 Vgl. BaFin (2014), S. 12. 
Tab. 4 Deskriptive Statistiken

\begin{tabular}{|c|c|c|c|c|c|c|}
\hline & & Mittelwert & Min & Max & SD & $N$ \\
\hline \multirow{3}{*}{$\begin{array}{l}\text { Anzahl der } \\
\text { Aufsichtsratsmitglieder }\end{array}$} & Deutschland & 9,7 & 3 & 20 & 5,3 & 49 \\
\hline & Österreich & 12,3 & 6 & 25 & 5,8 & 49 \\
\hline & Gesamt & 11,02 & 3 & 25 & 5,6 & 98 \\
\hline \multirow{3}{*}{$\begin{array}{l}\text { Davon } \\
\text { Arbeitnehmervertreter } \\
\text { (in \%) }\end{array}$} & Deutschland & 37,2 & 33,3 & 50 & 6,7 & 49 \\
\hline & Österreich & 27,3 & 0 & 36,4 & 12,9 & 49 \\
\hline & Gesamt & 32,2 & 0 & 50 & 11,4 & 98 \\
\hline \multirow{3}{*}{$\begin{array}{l}\text { Berichterstattung Diversität } \\
\text { (in \%) }\end{array}$} & Deutschland & 44 & 0 & 1 & 50 & 50 \\
\hline & Österreich & 41 & 0 & 1 & 50 & 49 \\
\hline & Gesamt & 42 & 0 & 1 & 50 & 99 \\
\hline \multirow{6}{*}{$\begin{array}{l}\text { Berichterstattung Diversität } \\
\text { im Zeitablauf (in \%) }\end{array}$} & 2009 & 16 & 0 & 1 & 37 & 19 \\
\hline & 2010 & 35 & 0 & 1 & 49 & 20 \\
\hline & 2011 & 50 & 0 & 1 & 49 & 20 \\
\hline & 2012 & 55 & 0 & 1 & 51 & 20 \\
\hline & 2013 & 55 & 0 & 1 & 51 & 20 \\
\hline & Gesamt & 42 & 1 & 1 & 50 & 99 \\
\hline \multirow{15}{*}{$\begin{array}{l}\text { Anteil Frauen am AR bei } \\
\text { börsennotierten } \\
\text { Gesellschaften (in \%) }\end{array}$} & Deutschland & & & & & \\
\hline & 2009 & 7,7 & 0 & 15 & 7,5 & 3 \\
\hline & 2010 & 7,7 & 0 & 15 & 7,5 & 3 \\
\hline & 2011 & 13 & 8 & 20 & 6,2 & 3 \\
\hline & 2012 & 22,3 & 20 & 25 & 2,5 & 3 \\
\hline & 2013 & 30,3 & 25 & 33 & 4,6 & 3 \\
\hline & Alle Jahre & 16,2 & 0 & 33 & 10,5 & 15 \\
\hline & Österreich & & & & & \\
\hline & 2009 & 13 & 13 & 13 & 0 & 1 \\
\hline & 2010 & 10 & 7 & 13 & 4,2 & 2 \\
\hline & 2011 & 11,5 & 10 & 13 & 2,1 & 2 \\
\hline & 2012 & 16,5 & 13 & 20 & 5 & 2 \\
\hline & 2013 & 10 & 0 & 20 & 14 & 2 \\
\hline & Alle Jahre & 12,1 & 0 & 20 & 6,2 & 9 \\
\hline & Gesamt & 14,7 & 0 & 33 & 9,2 & 24 \\
\hline
\end{tabular}

fallversicherung betreiben. ${ }^{45}$ Unter den Top-Ten der österreichischen Unternehmen sind alle (auch) in der Sparte Schaden-/Unfallversicherung tätig, die überwiegende Anzahl betreibt (auch) die Sparte Lebensversicherung. Insgesamt können alle als Kompositversicherer eingeordnet werden. In Deutschland ist die Sparte Lebensversicherung am häufigsten vertreten. Insgesamt können die untersuchten Top-Assekuranzen zur Hälfte als reine Lebens- bzw. Krankenversicherer klassifiziert werden, drei betreiben auch die Sparten Schaden bzw. Unfall (Zuordnung zu Kompositversicherer) und zwei sind als Rückversicherer zu qualifizieren.

Bezüglich der Mitgliederzahl der Aufsichtsräte (siehe hierzu und nachfolgend auch Tab. 4) lässt sich eine Diskrepanz zwischen deutschen und österreichischen

45 Vgl. Ebner und Ubl (2009), S. 3. 
Tab. 4 Deskriptive Statistiken (Fortsetzung)

\begin{tabular}{|c|c|c|c|c|c|c|}
\hline & & Mittelwert & Min & Max & SD & $N$ \\
\hline \multirow{3}{*}{$\begin{array}{l}\text { Anzahl der Frauen im AR } \\
\text { (absolut) }\end{array}$} & Deutschland & 1,7 & 0 & 5 & 1,3 & 49 \\
\hline & Österreich & 1,3 & 0 & 3 & 0,9 & 49 \\
\hline & Gesamt & 1,5 & 0 & 5 & 1,2 & 98 \\
\hline \multirow{3}{*}{$\begin{array}{l}\text { Aufsichtsräte ohne Frau } \\
\text { (in \%) }\end{array}$} & Deutschland & 22,4 & 0 & 1 & 42,2 & 49 \\
\hline & Österreich & 24,5 & 0 & 1 & 43,4 & 49 \\
\hline & Gesamt & 23,5 & 0 & 1 & 42,6 & 98 \\
\hline \multirow{15}{*}{$\begin{array}{l}\text { Anteil Frauen am AR } \\
\text { (in \%) }\end{array}$} & Deutschland & & & & & \\
\hline & 2009 & 14 & 0 & 33 & 11 & 9 \\
\hline & 2010 & 11,4 & 0 & 33 & 10 & 10 \\
\hline & 2011 & 14,7 & 0 & 50 & 14 & 10 \\
\hline & 2012 & 18,3 & 0 & 50 & 14 & 10 \\
\hline & 2013 & 20,7 & 0 & 50 & 16 & 10 \\
\hline & Alle Jahre & 15,9 & 0 & 50 & 13 & 49 \\
\hline & Österreich & & & & & \\
\hline & 2009 & 7,3 & 0 & 18 & 6 & 9 \\
\hline & 2010 & 10,1 & 0 & 18 & 7 & 10 \\
\hline & 2011 & 9,4 & 0 & 17 & 6 & 10 \\
\hline & 2012 & 14,4 & 0 & 33 & 10 & 10 \\
\hline & 2013 & 11,5 & 0 & 20 & 9 & 10 \\
\hline & Alle Jahre & 10,6 & 0 & 33 & 8 & 49 \\
\hline & Gesamt & 13,2 & 0 & 50 & 11 & 98 \\
\hline \multirow{3}{*}{$\begin{array}{l}\text { Frauenanteil bei } \\
\text { Arbeitnehmervertretern } \\
\text { (in \%) }\end{array}$} & Deutschland & 32 & 0 & 100 & 29 & 49 \\
\hline & Österreich & 19 & 0 & 100 & 19 & 41 \\
\hline & Gesamt & 26 & 0 & 100 & 26 & 90 \\
\hline \multirow{3}{*}{$\begin{array}{l}\text { Frauenanteil bei } \\
\text { Kapitalvertretern (in \%) }\end{array}$} & Deutschland & 7,7 & 0 & 50 & 11,4 & 49 \\
\hline & Österreich & 7,8 & 0 & 28,6 & 9,7 & 49 \\
\hline & Gesamt & 7,7 & 0 & 50 & 10,5 & 98 \\
\hline \multirow[t]{3}{*}{ Anzahl der AR-Sitzungen } & Deutschland & 3,3 & 2 & 6 & 1,5 & 44 \\
\hline & Österreich & 4,4 & 4 & 6 & 0,6 & 48 \\
\hline & Gesamt & 3,9 & 2 & 6 & 1,3 & 92 \\
\hline
\end{tabular}

Versicherungsunternehmen die gesamte Untersuchungsperiode hindurch erkennen: Im Durschnitt weisen die größten deutschen Versicherer 9,7 Aufsichtsratsmitglieder auf und österreichische Gesellschaften 12,3. Das Minimum der ganzen Grundgesamtheit lag bei 3 (DKV Deutsche Krankenversicherung AG, AXA Versicherung AG), das Maximum bei 25 Aufsichtsratsmitgliedern (S-Versicherung - Sparkassen Versicherung AG). Da die Zahlen die Personen inkl. Belegschaftsvertreter umfasst liegt sie sogar unter den gesetzlichen Höchstwerten (für Österreich nur für Kapitalvertreter 20, in Deutschland 21).

Der durchschnittliche prozentuelle Anteil der Arbeitnehmervertreter im Aufsichtsrat liegt für deutsche Versicherungsgesellschaften bei $37,2 \%$, bei den österreichischen Assekuranzen bei nur 27,3\%. Grund hierfür ist, dass bei zwei deutschen Versicherungsunternehmen - größenbedingt - der Aufsichtsrat zur Hälfte aus Ar- 
Tab. 4 Deskriptive Statistiken (Fortsetzung)

\begin{tabular}{|c|c|c|c|c|c|c|}
\hline & & Mittelwert & Min & Max & SD & $N$ \\
\hline \multirow{3}{*}{$\begin{array}{l}\text { AR-Vergütung gesamt in } \\
\text { Tsd. EUR }\end{array}$} & Deutschland & 591 & 22 & 2.321 & 670 & 49 \\
\hline & Österreich & 132 & 0 & 386 & 130 & 49 \\
\hline & Gesamt & 361 & 0 & 2.321 & 533 & 98 \\
\hline \multirow{3}{*}{$\begin{array}{l}\text { AR-Vergütung pro Kopf in } \\
\text { Tsd. EUR }\end{array}$} & Deutschland & 56 & 7 & 174 & 45 & 49 \\
\hline & Österreich & 15 & 0 & 39 & 13 & 49 \\
\hline & Gesamt & 36 & 0 & 174 & 39 & 98 \\
\hline \multirow[t]{3}{*}{ EKR (EGT/EK) in \% } & Deutschland & 29,2 & 3,7 & 70,1 & 15,8 & 50 \\
\hline & Österreich & 12,1 & $-25,9$ & 47,9 & 13 & 49 \\
\hline & Gesamt & 20,8 & $-25,9$ & 70,1 & 16,8 & 99 \\
\hline \multirow[t]{3}{*}{ EKR in \% nach Sparten } & $\begin{array}{l}\text { Lebens- und } \\
\text { Krankenversiche- } \\
\text { rer }\end{array}$ & 32,6 & 9,9 & 60,9 & 60,9 & 25 \\
\hline & $\begin{array}{l}\text { Komposit- } \\
\text { versicherung }\end{array}$ & 15,6 & $-25,9$ & 70 & 16,4 & 64 \\
\hline & Rückversicherer & 24,2 & 16,3 & 35 & 6,4 & 10 \\
\hline \multirow[t]{3}{*}{ Bilanzsumme in Mio. EUR } & Deutschland & 52.146 & 8.987 & 168.386 & 42.355 & 50 \\
\hline & Österreich & 6.188 & 1.497 & 15.672 & 3.905 & 49 \\
\hline & Gesamt & 29.400 & 1.497 & 168.386 & 37.918 & 99 \\
\hline \multirow{3}{*}{$\begin{array}{l}\text { Verrechnete Bruttopramien } \\
\text { in Mio. EUR }\end{array}$} & Deutschland & 8.186 & 3.176 & 25.541 & 6.015 & 50 \\
\hline & Österreich & 1.008 & 37 & 2.433 & 683 & 49 \\
\hline & Gesamt & 4.633 & 37 & 25.541 & 5.597 & 99 \\
\hline
\end{tabular}

Die Tabelle enthält neben den Mittelwerten den Minimal- sowie Maximalwert (Min bzw. Max), die Standardabweichung (SD) sowie die Anzahl der Beobachtungen (N)

beitnehmervertretern besteht (Allianz SE ${ }^{46}$ und Münchener Rück AG), wohingegen in zwei österreichischen Unternehmen kein Betriebsrat bestand (Vienna Insurance Group AG sowie Raiffeisen Versicherung AG) und somit keine Mitglieder vom Betriebsrat in den Aufsichtsrat entsandt wurden.

Es wurde ferner untersucht, ob über Diversität in den Geschäftsberichten berichtet wurde. Dazu wurden die Geschäftsberichte der Jahre 2009 bis 2013 aller Unternehmen auf die Informationen zum Thema „Diversität“ und „Frauen“ durchsucht. Dabei wird zwischen keinerlei Informationen und bereitgestellten Informationen zu Diversity differenziert (ja/nein, binäre Variable). Anzumerken ist, dass die Berichterstattung dabei sowohl qualitativer als auch quantitativer Natur sein kann. Unter qualitativen Informationen sind allgemeine, verbale Aussagen und Erläuterungen zu verstehen, wie zum Beispiel Angaben zu organisationalen Maßnahmen zur Steigerung von Diversität bzw. des Frauenanteils. Quantitative Informationen sind zahlenbasierte Darstellungen, wie Aussagen zum Anteil von Frauen in verschiedenen Führungsgremien. Insgesamt kommt die Untersuchung zu dem Ergebnis, dass sich in $42 \%$ der untersuchten Geschäftsberichten Informationen qualitativer und/ oder quantitativer Art zu Diversity bzw. Frauenförderung in Führungspositionen fin-

\footnotetext{
46 Die Allianz hat den in Deutschland gültigen Standard der Arbeitnehmer-Mitbestimmung bei der Umwandlung in eine SE übernommen. Vgl. Allianz (2007), o.S.
} 
den. Insgesamt ignorieren $58 \%$ das Thema vollkommen. Zu konstatieren ist, dass das Thema Diversität im Zeitablauf an Bedeutung gewonnen hat: 2009 geht weniger als ein Fünftel im Geschäftsbericht auf Diversity ein, 2013 legt mehr als die Hälfte darauf ein Augenmerk (s. dazu auch Tab. 4). Es zeigen sich hier ähnliche Ergebnisse für deutsche und österreichische Assekuranzen.

Zum Ende der Erhebung berichten die Versicherer zwar überwiegend über Diversity und insbesondere über Frauenförderung, aber nur die deutschen börsennotierten Unternehmen (bzw. jene, die einem börsennotierten Konzern angehören) nennen im Sinne des DCGK - konkrete Ziele und informieren über die Zielerreichung. Die Münchener Rück etwa gibt an: „Dabei sollen zu Beginn der nächsten Amtszeit (voraussichtlich 2014) mindestens $20 \%$ der Mitglieder und zu Beginn der darauffolgenden Amtszeit (voraussichtlich 2019) mindestens $30 \%$ der Mitglieder weiblich sein. “ ${ }^{47}$ Die Allianz SE informiert im Corporate Governance-Bericht: „Es wird ein Anteil von mindestens $25 \%$ Frauen im Aufsichtsrat angestrebt. Die Beteiligung von Frauen wird grundsätzlich als gemeinsame Verantwortung von Anteilseigner- und Arbeitnehmerseite gesehen. “48 „Kein österreichischer Versicherer - auch nicht die beiden börsennotierten Unternehmen - formulierte 2013 konkrete Ziele (solche werden vom Kodex auch nicht angeregt). “49

Betrachtet man die Anzahl von Frauen im Aufsichtsrat, so liegt dieser durchschnittlich bei 1,7 weiblichen Mitgliedern in deutschen und bei 1,3 in österreichischen Aufsichtsratsgremien. Es fällt dabei nicht nur die geringe Zahl pro Aufsichtsrat auf, sondern auch, dass rund einem Viertel (etwa $24 \%$ ) der untersuchten Aufsichtsräte überhaupt keine Frau als Mitglied angehört (s. Tab. 4, Variable „Aufsichtsräte ohne Frau (in \%)“). Keines der Unternehmen hatte im Untersuchungszeitraum eine weibliche Aufsichtsratsvorsitzende oder eine weibliche stellvertretende Aufsichtsratsvorsitzende. Ende 2013 war in drei österreichischen Assekuranzen keine Aufsichtsrätin vertreten (Uniqa Insurance Group AG, Generali Holding Vienna AG, Raiffeisen Versicherung AG) vertreten, in Deutschland waren zwei Kontrollgremien zur Gänze männlich (DKV Deutsche Krankenversicherung AG, AXA Versicherung AG). Die höchste Anzahl von Frauen im Aufsichtsrat hatte die Münchener Rück AG: Im Jahr 2013 gehörten 5 weibliche Mitglieder dem Kontrollgremium an (entsprach einem Anteil von $25 \%$ ).

Im Beobachtungszeitraum nimmt allerdings der Frauenanteil im Aufsichtsrat in den deutschen und österreichischen Gesellschaften zu. Durchschnittlich beträgt die Frauenquote in Deutschland in den Jahren 2009 bis 2013 rund 16\%. Über die Jahre hinweg stieg der Frauenanteil in den deutschen Gremien von rd. $14 \%$ im Jahr 2009 auf rund $21 \%$ im Jahre 2013 an. Damit liegen die deutschen Versicherer über dem mittleren Frauenanteil der größten deutschen Unternehmen in den Aufsichtsräten, dieser Betrug 2013 etwa 15\%. ${ }^{50}$ Die börsennotierten Unternehmen haben zuletzt sogar im Durchschnitt einen Anteil weiblicher Aufsichtsräte von über $30 \%$ (s. Tab. 4).

\footnotetext{
47 Munich Re (2013), S. 26.

48 Vgl. Geschäftsbericht Allianz SE (2013), S. 30.

49 Vgl. Uniqa Insurance Group AG (2013), S. 10.

50 Vgl. Holst und Kirsch (2014), S. 29.
} 


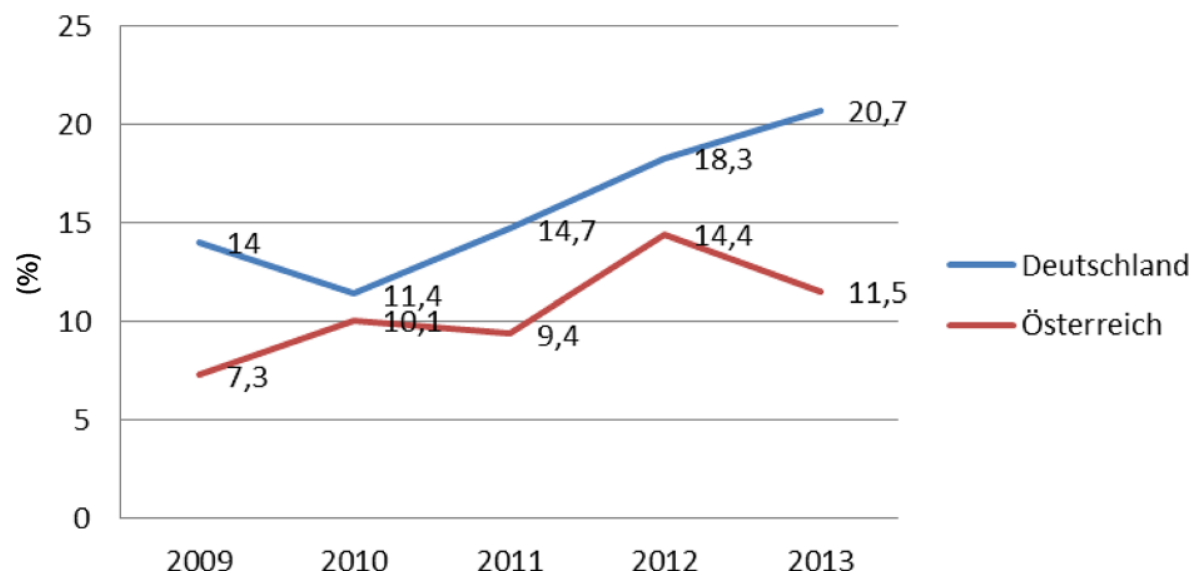

Abb. 1 Frauenanteil im Aufsichtsrat im Zeitablauf

In Österreich beträgt der durchschnittliche Frauenanteil bei den Kontrollorganen nur rund $11 \%$, wobei auch hier ein Anstieg von rund $7 \%$ im Jahr 2009 auf rund 12\% im Jahr $2013 \mathrm{zu}$ verzeichnen ist (s. dazu Abb. 1). Die börsennotierten Versicherer haben dabei keine höheren Frauenquoten (vgl. Tab. 4). Damit erzielt die Versicherungsbranche in Österreich zuletzt einen vergleichbaren Frauenanteil in den Boards wie die größten Unternehmen des Landes; der Durchschnitt betrug hier rd. $13 \% .{ }^{51}$ Deutsche Versicherer haben somit - gemessen anhand der Dimension Geschlecht - ein merklich höheres Ausmaß an Board-Diversität als österreichische.

Von Interesse ist auch, ob der Frauenanteil bei den Arbeitnehmervertretern höher als bei den Aktionärsvertretern ist. Sowohl in Deutschland als auch in Österreich zeigt sich, dass die Kapitalvertreter anteilsmäßig eine geringere Frauenquote aufweisen: Im Durchschnitt nominieren deutsche Aktionärsvertreter nur rd. $8 \%$ weibliche Kontrollorinnen, wohingegen der Anteil der weiblichen Arbeitnehmervertreterinnen bei rd. $32 \%$ liegt. Ein ähnliches Bild zeichnet sich für österreichische Versicherungen: Während die Aktionärsvertreter im Aufsichtsrat nur zu rd. $8 \%$ weiblich sind, beträgt der Frauenanteil der vom Betriebsrat entsendeten Mitglieder rd. 19\%. Insgesamt sind hier markante Unterschiede zu erkennen: Während die Arbeitnehmervertreter der Versicherungsgesellschaften etwa ein Viertel weibliche Aufsichtsräte entsendet, ist nicht einmal jedes zehnte Aufsichtsratsmitglied der Kapitalvertreter weiblich. Die Arbeitnehmerseite trägt somit maßgeblich zur Diversität bei.

Legt man das Augenmerk auf die aus den Geschäftsberichten entnommenen Zahlen der Aufsichtsratssitzungen, so zeigt sich grundsätzlich ein durchschnittlicher Wert von rd. 4 Sitzungen pro Jahr (es konnten nur die Daten aus 92 Geschäftsberichten verarbeitet werden, der Rest berichtete nicht darüber). In Deutschland hielt die Hälfte der untersuchten Unternehmen in den Jahren 2009 bis 2013 überwiegend bloß zwei Sitzungen ab. Österreichische Versicherer hielten merklich mehr Sitzungen ab (durchschnittlich rd. eine zusätzliche Sitzung pro Jahr).

51 Vgl. Arbeiterkammer Wien (2014), S. 3. 
Des Weiteren interessieren die Höhe bzw. die Angemessenheit der Aufsichtsratvergütung der größten Versicherungsgesellschaften. Hier zeigt sich, dass die Vergütung der deutschen Aufsichtsratsmitglieder signifikant höher ausfällt. Die mittlere Vergütung des gesamten Aufsichtsrats in Deutschland belief sich auf jährlich 591 Tsd. EUR, jene des österreichischen auf 132 Tsd. Euro. Während in Österreich zwei Versicherer ihren Kontrolleuren im gesamten Untersuchungszeitraum keinerlei Vergütung für ihre Tätigkeit bezahlten ${ }^{52}$, war die geringste jährliche Gesamtvergütung der deutschen Aufsichtsräte in der Untersuchung 22 Tsd. Euro. Die höchste Aufsichtsratsvergütung in Deutschland belief sich auf rd. 2,3 Mio. $€$ (Münchener Rück AG im Jahr 2013), in Österreich betrug diese 386 Tsd. Euro (Vienna Insurance Group AG im Jahr 2013).

Ein ähnliches Bild zeigt sich, wenn die Vergütung der Ratsmitglieder pro Kopf betrachtet wird. Anzumerken ist, dass eine detaillierte Analyse der Vergütungskomponenten (feste und variable Bestandteile) sowie der Vergütung des Vorsitzenden bzw. des Stellvertreters (diese erhalten regelmäßig höhere Vergütungen) aufgrund der überwiegend fehlenden (bzw. nicht individualisiert offengelegten) Informationen in den Geschäftsberichten nicht möglich war. Dabei wurde die durchschnittliche Vergütung in Österreich pro Aktionärsvertreter berechnet, während in Deutschland die Gesamtbezüge durch die Anzahl aller Mitglieder dividiert wurde. ${ }^{53}$ Ein Mitglied einer deutschen Top-Versicherungsgesellschaft erhielt im Durchschnitt rd. 56 Tsd. Euro jährliche Vergütung, ein österreichisches nur rund 15 Tausend, wobei hier größere Schwankungsbreiten zu beobachten sind (Maximalvergütung pro Kopf in Deutschland 174 Tsd. Euro (Allianz SE im Jahr 2012), in Österreich 39 Tsd. Euro (Vienna Insurance Group AG im Jahr 2013)). Im internationalen Vergleich ist damit die Entlohnung für die Kontrolleure in der deutschen - und insbesondere in der österreichischen - Versicherungsbranche als unterdurchschnittlich anzusehen. ${ }^{54}$

Betrachtet man die Eigenkapitalrendite (EGT dividiert durch Eigenkapital) so liegen die Mittelwerte im Zeitraum 2009 bis 2013 in Deutschland bei rund 29\% und in Österreich bei rund $12 \%$. Trotz der schwierigen Berechenbarkeit (Zurechnungsproblematik einzelner Posten, Finanzierungseinfluss) soll die einheitliche Berechnung der Werte dennoch zur Aussagefähigkeit bezüglich der Profitabilität wesentlich beitragen. Die deutschen Assekuranzen sind im Beobachtungszeitraum deutlich profitabler als die österreichischen. Durchschnittlich weisen die Lebens- bzw. Krankenversicherer, gefolgt von den Rückversicherern, die höchste Profitabilität auf. Die geringste Rentabilität verzeichneten die Kompositversicherer. Allerdings schwankt die Performance hier durchaus sehr stark (s. Tab. 4).

Im Durchschnitt sind die deutschen Versicherungen signifikant größer als die österreichischen Assekuranzen. Die mittlere Bilanzsumme der deutschen Gesellschaften beträgt 52.146 Mio. $€$, jene der österreichischen 6.188 Mio. €. Im Durchschnitt

\footnotetext{
52 Dabei handelt es sich um die Raiffeisen Versicherung AG sowie um die Ergo Versicherung AG. Hierbei ist allerdings die Tätigkeit im Konzernverbund als wichtiger Einflussfaktor anzuführen.

53 In Deutschland gibt es für Aktionärs- und Arbeitnehmervertreter grundsätzlich eine Gleichbehandlung bei ihrer Vergütung. Vgl. dazu Evers (2009), S. 378. In Österreich üben die Arbeitnehmervertreter ihre Tätigkeit ehrenamtlich - ohne Vergütung - aus; vgl. § 110 Abs 3 Satz 1 öArbVG.

54 Vgl. dazu bspw. die Ergebnisse der Untersuchung der Hay Group (2013), S. 3 ff.
} 
belaufen sich die verrechneten Bruttoprämien der deutschen Versicherungen auf 8.186 Mio. $€$ jährlich und die der österreichischen auf 1.008 Mio. $€$.

\subsection{Korrelationsanalyse}

Ausgehend von den dargestellten Daten sollen nun weitergehende Fragestellungen entsprechend den obigen Zielsetzungen beantwortet werden. Eine Korrelationsanalyse zeigt folgende Zusammenhänge auf (s. Korrelationsmatrix in Tab. 5). Dabei sind Korrelationen auf dem $5 \%$-Signifikanzniveau fettgedruckt.

Die Analyse zeigt, dass größere Aufsichtsräte eine merklich höhere Anzahl von Aufsichtsratssitzungen abhalten, eine höhere Gesamtvergütung erhalten (jedoch nicht pro Kopf) und börsennotierte Versicherungsunternehmen signifikant größere Gremien eingerichtet haben. Bei größeren Kontrollgremien ist die Wahrscheinlichkeit signifikant höher, dass (zumindest) eine Frau als Kontrollorgan fungiert (s. dichotome Variable ,(2) Frau(en) im Aufsichtsrat“; die Variable nimmt den Wert 1 an, wenn zumindest eine Frau im Aufsichtsrat vertreten ist, andernfalls ist sie 0). Zwischen Größe des Aufsichtsrats und Performance ist kein Zusammenhang feststellbar.

Der Frauenanteil am Aufsichtsrat ist mit der Unternehmensgröße (sowohl gemessen anhand der Bilanzsumme als auch gemessen anhand der verrechneten Bruttoprämien) signifikant positiv korreliert. Dieser Zusammenhang ist im Einklang mit den Ergebnissen bisheriger Studien. ${ }^{55}$ Unternehmen mit einem höheren Anteil an Frauen berichten auch verstärkt über (Gender-)Diversität. Damit legen die Ergebnisse dieser Untersuchung nahe, dass die größten - und insbesondere die börsennotier-

Tab. 5 Korrelationsmatrix

\begin{tabular}{|c|c|c|c|c|c|c|c|c|c|c|c|}
\hline & (1) & (2) & (3) & (4) & (5) & (6) & (7) & (8) & (9) & (10) & (11) \\
\hline (1) Größe AR & 1 & - & - & - & - & - & - & - & - & - & - \\
\hline $\begin{array}{l}\text { (2) Frau(en) im } \\
\text { AR }\end{array}$ & $\mathbf{0 , 5 3}$ & 1 & - & - & - & - & - & - & - & - & - \\
\hline (3) Frauenanteil & 0,09 & 0,67 & 1 & - & - & - & - & - & - & - & - \\
\hline (4) Diversität & 0,24 & 0,19 & 0,24 & 1 & - & - & - & - & - & - & - \\
\hline (5) Sitzungen & $\mathbf{0 , 3 5}$ & 0,08 & $-0,01$ & 0,25 & 1 & - & - & - & - & - & - \\
\hline (6) Vergütung & $\mathbf{0 , 2 9}$ & 0,22 & 0,22 & 0,35 & $\mathbf{0 , 4 1}$ & 1 & - & - & - & - & - \\
\hline $\begin{array}{l}\text { (7) Vergütung } \\
\text { pro Kopf }\end{array}$ & $-0,02$ & 0,05 & 0,17 & 0,28 & $\mathbf{0 , 2 1}$ & 0,87 & 1 & - & - & - & - \\
\hline (8) EKR & $-0,07$ & $-0,01$ & 0,13 & 0,17 & $-0,54$ & $-0,07$ & 0,02 & 1 & - & - & - \\
\hline $\begin{array}{l}\text { (9) Bilanzsum- } \\
\text { me }\end{array}$ & $-0,04$ & 0,22 & 0,20 & 0,21 & $-0,08$ & $\mathbf{0 , 5 3}$ & 0,49 & 0,26 & 1 & - & - \\
\hline $\begin{array}{l}\text { (10) Bruttoprä- } \\
\text { mien }\end{array}$ & 0,05 & 0,16 & 0,32 & $\mathbf{0 , 3 0}$ & 0,03 & $\mathbf{0 , 5 8}$ & 0,44 & 0,37 & $\mathbf{0 , 7 0}$ & 1 & - \\
\hline $\begin{array}{l}\text { (11) Börsenno- } \\
\text { tiert }\end{array}$ & 0,26 & 0,15 & 0,07 & 0,47 & 0,55 & 0,73 & 0,65 & $-0,26$ & 0,22 & $\mathbf{0 , 3 1}$ & 1 \\
\hline
\end{tabular}

Fettgedruckte Korrelationen sind zum $5 \%$-Niveau signifikant

55 s. bspw. Adams und Ferreira (2009), S. 295. 
ten - Versicherungsunternehmen auf Diversität ein besonderes Augenmerk legen. Sie entsprechen damit den erhöhten Transparenzanforderungen des Kapitalmarktes. Bei börsennotierten Unternehmen hat der Aufsichtsrat i. d. R. die Interessen einer breit(er) gestreuten Eigentümerschaft (Streubesitz) zu vertreten. Auch andere Studien belegen, dass international ausgerichtete (börsennotierte) Unternehmen vermehrt Diversity-Management-Konzepte implementieren. ${ }^{56}$ Eine Rolle könnten dabei auch institutionelle Eigentümer spielen, die „Shareholder Activism“ in Richtung Diversität betreiben. ${ }^{57}$ Versicherer, die auf Diversität abstellen, vergüten ihre Aufsichtsratsmitglieder höher. Dies zeigt sich auch in der Studie von Adams und Ferreira (2009), wobei die Autoren hier zeigen, dass der Frauenanteil mit einer höheren aktienbasierten Entlohnung sowie Gesamtvergütung der Mitglieder einhergeht. Adams und Ferreira argumentieren, dass die höhere performancebezogenen Entlohnung zu einer Angleichung der Interessen von Boardmitglieder und Eigentümer führt und daher im Sinne der Shareholder-Value-Orientierung ist. ${ }^{58}$ Es zeigt sich hier kein signifikanter Zusammenhang zwischen dem Anteil der weiblichen Aufsichtsräte bzw. der Berücksichtigung von Diversität und der Profitabilität.

Erfolgreichere Versicherungsunternehmen (gemessen anhand der Eigenkapitalrentabilität) halten signifikant weniger Aufsichtsratssitzungen ab. Versicherer, bei denen der Aufsichtsrat häufiger tagt, haben größere Gremien eingerichtet. Damit sind die Ergebnisse in der Versicherungswirtschaft völlig im Einklang mit der vielzitierten Studie von Vafeas (1999). ${ }^{59}$

Die Aufsichtsratsvergütung pro Kopf ist mit der Unternehmensgröße hochsignifikant korreliert (sowohl anhand der Bilanzsumme als auch anhand der verrechneten Prämien) und bestätigt, dass die Größe des Unternehmens der wichtigste Bestimmungsfaktor für die Vergütung der Mitglieder ist. ${ }^{60}$ Mit dem Erfolg des Versicherers zeigt sich in der Stichprobe kein signifikanter Zusammenhang. Auch stehen die Bezüge pro Kopf nicht mit der Größe des Aufsichtsrats (Mitgliederanzahl) in Verbindung. Börsennotierte Gesellschaften zahlen ihren Kontrolleuren signifikant höhere Vergütungen.

Trotz der Erkenntnisse, die der Beitrag über Aufsichtsratsmerkmale für die Versicherungswirtschaft liefert, wird abschließend auf die Limitationen der Untersuchung verwiesen. Die Ergebnisse basieren auf einer Stichprobe der 20 größten Unternehmen in Deutschland und Österreich in einem Fünf-Jahres-Zeitraum. Hier wäre eine Ausweitung auf eine größere Anzahl von Unternehmen in der Versicherungsbranche von Interesse.

\footnotetext{
56 Vgl. dazu auch die Ergebnisse in Lančarič et al. (2015), S. 1162 ff.

57 Vgl. Farrell und Hersch (2005), S. 89.

58 Vgl. Adams und Ferreira (2009), S. 303.

59 Vgl. Vafeas (1999).

60 Vgl. dazu die Ausführungen Evers (2009), 355 ff.
} 


\section{Zusammenfassung}

Der Beitrag geht auf Aspekte der Corporate Governance und des Aufsichtsrats von deutschen und österreichischen Versicherungen ein. Dazu wurden im empirischen Teil ausgewählte Merkmale der größten deutschen und österreichischen Versicherungsgesellschaften im Zeitraum 2009 bis 2013 näher untersucht.

Österreichische Versicherer haben größere Gremien (eine höhere Anzahl von Aufsichtsratsmitgliedern) als die deutschen. Der Anteil der Arbeitnehmervertreter im Aufsichtsrat ist in Deutschland merklich höher.

Es zeigt sich, dass in den untersuchten Versicherungen Frauen im Aufsichtsrat klar unterrepräsentiert waren. Rd. ein Viertel der Gesellschaften hatte keine Frau im Aufsichtsrat. Im Zeitablauf erhöhte sich nicht nur die Berichterstattung über Diversität, sondern auch der Frauenanteil merklich; dieser beträgt zum Ende der Erhebung für deutsche Assekuranzen $21 \%$. Die börsennotierten deutschen Versicherungsgesellschaften haben 2013 einen höheren Anteil weiblicher Vertreter von durchschnittlich rd. $30 \%$, womit die Frauenquote hier schon 2013 erfüllt war. Dazu könnte auch bereits die Diskussion um Einführung gesetzlicher Frauenquoten im Vorfeld geführt haben. In Österreich ist der Frauenanteil in den letzten Jahren ebenfalls angestiegen, beträgt zuletzt aber nur rd. $11 \%$. Österreich hat diesbezüglich starken Aufholbedarf. In beiden Ländern trägt die Arbeitnehmerseite maßgeblich zur Gender-Diversität bei und entsendet sehr viel häufiger weibliche Vertreter in den Aufsichtsrat als die Kapitalgeberseite.

Die Vergütung der Kontrollorgane ist in Deutschland signifikant höher als in Österreich, wobei dies vor allem auf die Größe der Versicherer zurückgeführt werden kann. Im Vergleich zu anderen Studien ist die Vergütung der Gremienmitglieder in der Versicherungsbranche als moderat einzustufen.

Die Rentabilität der deutschen Gesellschaften ist höher als jene der österreichischen Versicherer, unterscheidet sich jedoch vor allem nach Sparten. In der Untersuchung zeigt sich kein Zusammenhang von Diversitätsaspekten mit der Performance. Die Ergebnisse legen den Schluss nahe, dass in der Versicherungsbranche vor allem die Unternehmensgröße und die Kapitalmarktpräsenz Faktoren für DiversityManagement sind.

Danksagung Wir danken Frau Tamara Fuchs für die Bereitstellung der Rohdaten für die Analyse.

Open Access Dieser Artikel wird unter der Creative Commons Namensnennung 4.0 International Lizenz (http://creativecommons.org/licenses/by/4.0/deed.de) veröffentlicht, welche die Nutzung, Vervielfältigung, Bearbeitung, Verbreitung und Wiedergabe in jeglichem Medium und Format erlaubt, sofern Sie den/die ursprünglichen Autor(en) und die Quelle ordnungsgemäß nennen, einen Link zur Creative Commons Lizenz beifügen und angeben, ob Änderungen vorgenommen wurden.

Funding Open access funding provided by Johannes Kepler University Linz.

\section{Literatur}

Adams, R.B., Ferreira, D.: Women in the boardroom and their impact on governance and performance. J. Financ. Econ. 94(2), 291-309 (2009) 
Allianz, S.E.: Erste reguläre Wahl zum Aufsichtsrat der Allianz SE (2007). https://www.allianz.com/de/ presse/news/unternehmen/corporate_governance/news_2007-05-02.html/, Accessed 8 May 2017

Arbeiterkammer Wien: Frauen.Management.Report.2014, Frauen in Geschäftsführung \& Aufsichtsrat in den Top 200 und börsennotierten Unternehmen (2014). http://www.forschungsnetzwerk.at/ downloadpub/2014_AK_Frauen_Management_Report_2014.pdf, Accessed 8 May 2017

BaFin, Bundesanstalt für Finanzdienstleistungsaufsicht, Statistik der Bundesanstalt für Finanzdienstleistungsaufsicht: Erstversicherungsunternehmen und Pensionsfonds (2014). https://www.bafin.de/ SharedDocs/Downloads/DE/Statistik/Erstversicherer/dl_st_14_erstvu_pf_va_xls.html, Accessed 8 May 2017

Bermig, A., Frick, B.: Determinanten der „Übergröße“ deutscher Aufsichtsräte. Schmollers Jahrb. 131(1), 169-194 (2011)

Bhagat, S., Black, B.: The non-correlation between board independence and long-term firm performance. J. Corp. Law 27, 231 (2002)

Carter, D.A., Simkins, B.J., Simpson, W.G.: Corporate governance, board diversity, and firm value. Financ. Rev. 38(1), 33-53 (2003)

Cheng, S.: Board size and the variability of corporate performance. J. Financ. Econ. 87, 157-176 (2008)

Dalton, D.R., Daily, C.M., Ellstrand, A.E., Johnson, J.L.: Meta-Analytic reviews of board composition, leadership structure and financial performance. Strateg. Manage. J. 19(3), 269-290 (1998)

Deutscher Corporate Governance Kodex: Deutscher Corporate Governance Kodex (2010). http://www. corporate-governance-code.de/index.html (Created 26 May 2010), Accessed 8 May 2017

Drygala, T.: § 110. In: Schmidt, K., Lutter, M. (eds.) Aktiengesetz, Kommentar, 3rd edn. vol. 1, pp. 1701-1708. Schmidt, Köln (2015a)

Drygala, T.: $\S \S 95,96$. In: Schmidt, K., Lutter, M. (eds.) Aktiengesetz, Kommentar, 3rd edn. vol. 1, pp. 1481-1516. Schmidt, Köln (2015b)

Ebner, G., Ubl, E.: Die österreichische Versicherungswirtschaft aus Sicht der Finanzmarktstabilität - eine Analyse der Jahre 2002 bis 2008. Finanzmarktstabilitätsbericht 18(1), 78-95 (2009)

Eisenberg, T., Sundgren, S., Wells, M.: Larger board size and decreasing firm value in small firms. J. Financ. Econ. 48(1), 187-223 (1998)

Eling, M., Pankoke, D.A.: Deutscher Aufsichtsrat versus Schweizer Verwaltungsrat, Systematischer Abgleich und Evaluation der zentralen Kontroll- und Leitungsgremien im deutschsprachigen Versicherungsbereich. Z. Gesamte Versicherungswiss. 103(2), 137-165 (2014)

Erhardt, N.L., Werbel, J.D., Shrader, C.B.: Board of director diversity and firm financial performance. Corporate Governance: An International Review 11(2), 102-111 (2003)

Evers, H.: Vorstands- und Aufsichtsratsvergütung. In: Hommelhoff, P., Hopt, K.J., v. Werder, A. (eds.) Handbuch Corporate Governance, 2nd edn., pp. 349-387. Schmidt, Stuttgart (2009)

Farrell, K.A., Hersch, P.L.: Additions to corporate boards: the effect of gender. J. Corp. Finance 11(1), 85-106 (2005)

Gardenswartz, L., Rowe, A.: Diverse Teams at Work Capitalizing on the Power of Diversity. Soc For Human Resource Mgmt, Alexandria (2003)

Guserl, R., Pernsteiner, H.: Finanzmanagement: Grundlagen - Konzepte - Umsetzung, 2nd edn. Springer Gabler, Wiesbaden (2015)

Harrigan, K.R.: Numbers and positions of women elected to corporate boards. Acad. Manage. J. 24(3), 619-625 (1981)

Hay Group: Non-executive directors in Europe, casting light on pay practices, structures and diversity of leading European companies (2013). https://www.haygroup.com/downloads/pt/hay_group_non_ executive_directors_in_europe_2013.pdf, Accessed 8 May 2017

Heidemann, J., Landherr, A., Müller, A.L.: Berichterstattung über Frauen in Führungspositionen: eine Analyse der DAX 30-Unternehmen. Schmalenbachs Z. Betriebswirtsch. Forsch. 65(5), 488-512 (2013)

Hermalin, B.E., Weisbach, M.S.: The effects of board composition and direct incentives on firm performance. Financ. Manage 20(4), 101-112 (1991)

Hölscher, R., Dähne, C.: Anforderungen an Aufsichtsorgane von Kreditinstituten im Rahmen des dualen Führungssystems. In: Paetzmann, K., Schöning, S. (eds.) Corporate Governance von Kreditinstituten, pp. 271-295. Schmidt, Berlin (2014)

Holst, E., Kirsch, A.: Frauen in Vorständen großer Unternehmen in Deutschland noch immer die Ausnahme - moderat steigende Anteile in Aufsichtsräten. DIW Wochenber. 81(3), 19-32 (2014)

Horváth, R., Spirollari, P.: Do the board of directors' characteristics influence firm's performance? The U.S. evidence. Prague Econ. Pap. 4, 470-486 (2012)

Hüffer, U., Koch, J.: Aktiengesetz, 12th edn. Beck'sche Kurzkommentare. Beck, München (2016)

Kalss, S.: Frauen im Aufsichtsrat - mehr als nur ein Schlagwort. Aufsichtsrat Aktuell 1, 5-9 (2011) 
Kalss, S., Schimka, M.: Qualifikationsanforderungen der AR-Mitglieder. In: Kalss, S., Kunz, P. (eds.) Handbuch für den Aufsichtsrat, 2nd edn., pp. 75-108. Facultas, Wien (2016)

Kalss, S., Wendt, D.H.: Besonderheiten des Aufsichtsrats in Versicherungsunternehmen. In: Kalss, S., Kunz, P. (eds.) Handbuch für den Aufsichtsrat, 2nd edn., pp. 1287-1306. Facultas, Wien (2016)

Kalss, S.: § 94. In: Doralt, P., Nowotny, C., Kalss, S. (eds.) Kommentar zum Aktiengesetz, 2nd edn. vol. 1, pp. 1271-1280. Linde, Wien (2012)

Koeberle-Schmid, A.: Professionelle Aufsichtsgremien: Aufgaben, Typen und Ausgestaltung. In: Koeberle-Schmid, A., Fahrion, H.-J., Witt, P. (eds.) Family Business Governance: Erfolgreiche Führung von Familienunternehmen, 2nd edn., pp. 120-154. Schmidt, Berlin (2012)

Krell, G., Riedmüller, B., Sieben, B., Vinz, D.: Einleitung-Diversity Studies als integrierende Forschungseinrichtung. In: Krell, G., Riedmüller, B., Sieben, B., Vinz, D. (eds.) Diversity Studies - Grundlagen und disziplinäre Ansätze, pp. 7-16. Peter Lang, New York (2007)

Lančarič, D., Chebeň, J., Savov, R.: Factors influencing the implementation of diversity management in business organisations in a transition economy: the case of Slovakia. Econ. Res. Ekon. Istraz. 28(1), 1162-1184 (2015)

McKinsey \& Company: Diversity matters (2014). https//web.duke.edu/equity/toolkit/documents/ Diversity Matters.pdf, Accessed 20 June 2016

Mensi-Klarbach, H.: Diversity und Diversity Management - die Business Case Perspektive: eine kritische Analyse. Schriftenreihe innovative betriebswirt-schaftliche Forschung und Praxis, vol. 258. Kovač, Hamburg (2010)

Munich Re: Konzerngeschäftsbericht 2013, https://www.munichre.com/site/corporate/get/params_E154004493/1388740/302-08162_de.pdf, Accessed 8 May 2017

Pernsteiner, H.: Zur Dauer von Aufsichtsratsmitgliedschaften von österreichischen börsennotierten Unternehmen. In: Hofer, K.M., Roithmayr, F. (eds.) Marketingaspekte, FS Gerhard Wührer, pp. 193-207. Trauner, Linz (2015)

Sepheri, P.: Diversity and Managing Diversity - Verständnisfragen, Zusammenhänge. In: Peters, S., Bensel, N. (eds.) Frauen und Männer im Management-Diversity in Diskurs und Praxis, 2nd edn., pp. 121-142. Gabler, Wiesbaden (2002)

Simon, W.: Moderne Managementkonzepte von A-Z. Gabal, Offenbach (2002)

Smith, N., Smith, V., Verner, M.: Do women in top management affect firm performance? A panel study of 2,500 Danish firms. Int. J. Productivity and Performance Management, 55(7), 569-593 (2006)

Strasser, R.: $§ \$ 98,99$. In: Jabornegg, P., Strasser, R. (eds.) Kommentar zum Aktiengesetz, 5th edn. vol. 2, Manz, Wien (2010)

Süß, S., Kleiner, M.: Diversity Management - Verbreitung in der deutschen Unternehmenspraxis und Erklärungen aus Neo-Institutionalistischer Perspektive. In: Krell, G., Wächer, H. (eds.) Diversity Management-Impulse aus der Personalforschung, pp. 57-80. Rainer Hampp, Mering (2006)

Uniqa Insurance Group AG: Jahresfinanzbericht 2013, http://www.uniqagroup.com/gruppe/versicherung/ media/files/UNIQA_Jahresfinanzbericht_2013.pdf, Accessed 8 May 2017

Vafeas, N.: Boardmeeting frequency and firm performance. J. Financ. Econ. 53(1), 113-142 (1999)

Versicherungsverband Österreich, Verband der Versicherungsunternehmen Österreichs VVO: Jahresbericht 2013 (2013). http://www.vvo.at/vvo/vvo.nsf/sysPages/x14104A57C6BD6C60C1257CE100438 EAC/\$file/Jahresbericht_2013.pdf, Accessed 8 May 2017

Watson, W.E., Kumar, K., Michaelsen, L.K.: Cultural diversity's impact on interaction processes and performance: comparing homogenous and diverse task groups. Acad. Man. J. 36(3), 590-602 (1993)

Welge, M.K., Eulerich, M.: Corporate Governance Management - Theorie und Praxis der guten Unternehmensführung. Gabler, Wiesbaden (2012)

Yermack, D.: Higher Market valuation of companies with a small board of directors. J. Financ. Econ. 40(2), 185-212 (1996) 